\title{
Extended-field intensity-modulated radiotherapy and dosage boost for lymph node metastasis are beneficial for the prognosis of FIGO IIICr and IVA cervical cancer patients with positive regional lymph nodes
}

Yuhua Zhao

Guangzhou University of Chinese Medicine

\section{Gong Li}

Guangzhou University of Chinese Medicine Second Affiliated Hospital (Guangdong Provincial Hospital of Chinese Medicine )

Lei Gao ( $\nabla$ gzgaolei@126.com )

https://orcid.org/0000-0002-4239-7718

\section{Research}

Keywords: FIGO staging, IIICr and IVA, cervical cancer, positive PALNs, extended-field intensity-modulated radiotherapy, simultaneously integrated boost-IMRT, sequential boost-IMRT

Posted Date: March 13th, 2020

DOl: https://doi.org/10.21203/rs.3.rs-17075/v1

License: (c) (1) This work is licensed under a Creative Commons Attribution 4.0 International License. Read Full License 


\section{Abstract}

Background: This study aimed to evaluate the therapeutic efficacy of extended-field intensity-modulated radiotherapy (EF-IMRT) and dosage boost for positive lymph nodes, prognostic factors, treatment failure, and toxicity for Federation of Gynecology and Obstetrics (FIGO) stage IIICr and IVA cervical cancer patients with positive regional lymph nodes.

Methods: We retrospectively evaluated 34 patients with stage IIICr and IVA who had received treatment in our institute between 2013 and 2016. Patients with stage IVA cervical cancer who had been enrolled in the analysis all had positive regional lymph nodes (pelvic or/and para-aortic). All 34 patients were treated with EF-IMRT and simultaneously integrated boost-IMRT (SIB-IMRT) for lymph node metastasis with concurrent chemotherapy and brachytherapy. Positive regional lymph nodes (short-axis diameter $\geq 5 \mathrm{~mm}$ in computed tomography [CT] or magnetic resonance imaging [MRI]) remaining after SIB-IMRT were then treated with sequential boost-IMRT (SeB-IMRT). The prognostic factors for overall survival (OS); diseasefree survival (DFS); local control rate (LCR); regional control rate (RCR); distant metastasis-free survival (DMFS), including age, FIGO stage, pretreatment hemoglobin (HB) level, tumor size, para-aortic lymph node (PALN) metastasis, point A equivalent dose in 2-Gy fractions (EQD2 dose), concurrent chemotherapy, and adjuvant chemotherapy cycles, were analyzed.

Results: Complete response (CR) was achieved in 31 (91.2\%) patients with acceptable adverse effects. Notably, the three-year OS, DFS, LCR, RCR, DMFS for these patients were $73.5 \%, 70.6 \%, 88.1 \%, 87.9 \%$, and $81.6 \%$, respectively. In particular, the three-year OS, DFS, LCR, RCR, and DMFS of patients with positive PALNs was $41.7 \%, 33.3 \%, 65.6 \%, 72.2 \%$, and $60.2 \%$, respectively. The corresponding values in patients without positive PALNs were $90.9 \%, 90.9 \%, 100 \%, 95.5 \%$, and $90.9 \%$, respectively.

Conclusions: Our study suggested that the EF-IMRT and nodal dosage boost decreased regional node failure and that patients with stage IIIC1r and IVA cervical cancer without PALN metastasis who received EF-IMRT and SIB-IMRT with or without SeB-IMRT had a significant survival advantage in terms of the DFS and OS.

\section{Background}

Cervical cancer is one of the most common cancers among women worldwide. Despite the continuous promotion and application of screening technologies, the incidence of cervical cancer remains high, with approximately 569,800 new cases and 311,400 deaths in 2018 [1]. Patients with cervical cancer stage III and IV have a poor prognosis, especially in cases where there is lymph node involvement [2]. Lymph node metastasis predicts poor survival, and positive para-aortic lymph nodes (PALNs) has been reported to have a more negative impact on survival than positive PLN [5]. To highlight the prognostic significance of lymph node metastasis, the 2018 version of International Federation of Gynecology and Obstetrics (FIGO) staging system classifies pelvic and PALN involvement as IIIC1 and IIIC2 for patients with no distant metastasis [24]. NCCN guidelines recommend pelvic external beam radiation therapy (EBRT) with 
concurrent chemotherapy and brachytherapy as standard therapy for LACC patients with positive low true pelvic nodes [3]. However, for stage III-IVA patients, especially those with positive PLNs (negative PALNs) who have high risk of PALN involvement $[17,18]$, pelvic radiotherapy may not completely eradicate the microscopic and subclinical lesions in para-aortic lymph nodes [6]. In our institute, we administered extended-field intensity-modulated radiotherapy (EF-IMRT) addressing the para-aortic region and intensive nodal boost to IIICr and IVA patients with positive PLNs regardless of the involvement of PALNs. IMRT has been reported to improve the sparing of at-risk organs [13], we hypothesized that EF-IMRT and intensive nodal boost would safely improve lymph node control and the OS and DFS in IIICr and IVA patients with positive regional lymph nodes. In the present study, we retrospectively analyzed the data of 34 FIGO grades IIICr and IVA cervical cancer patients with positive lymph nodes to evaluate the therapeutic efficacy, treatment failure, toxicity, and prognostic factors of EF-IMRT and intensive nodal boost.

\section{Method}

\section{Patient selection and data collection}

This retrospective review was conducted at the Radiation Oncology Department, Guangdong Provincial Hospital of Chinese Medicine. We reviewed the medical records of patients treated from 2013 to 2016. Based on the 2018 version of the FIGO staging system, we reclassified disease stage of the enrolled patients according to their medical records. The inclusion criteria were patients with biopsy-proven squamous cell carcinoma of the cervix graded with FIGO (2018) as stages IIICr and IVA. Enrolled patients received definitive chemoradiotherapy and image-guided adaptive brachytherapy with a curative aim. Patients with other pathological types of carcinoma, those undergoing hysterectomy, those with a history of previous malignancy, and those with an Eastern Cooperative Oncology Group (ECOG) score $\geq 2$ were excluded from the analysis. The workup before the primary treatment included comprehensive medical history, a gynecological examination, cervical biopsy implemented through colposcopy if necessary, chest X-ray, abdominopelvic magnetic resonance imaging (MRI) or computed tomography (CT), complete blood cell count, and squamous cell carcinoma (SCC)-associated antigen and blood biochemical testing. In some cases, image examination supported by 18 -fluorodeoxyglucose positron emission tomography (FDG-PET) was used. Patients underwent all the pretreatment workup to obtain diagnostic information. For cases with suspected stage IVA cancer, rectal and bladder invasion were confirmed by biopsy via cystoscopy and proctoscopy.

Lymph nodes with a short axial diameter of $>10 \mathrm{~mm}$ as visualized in CT or MRI images were defined as metastatic. In patients who had been examined by PET, nodes with a more avid FDG uptake than the adjacent background were considered positive.

\section{Radiotherapy}


All the patients who had been enrolled in this study received EF-IMRT, which consisted of seven coplanar $6 \mathrm{MV}$ photon fields, followed by high-dose-rate intracavitary brachytherapy. The radiation field for EBRT in this study was an extended field, including the standard pelvic radiation field and para-aortic region up to the level of the renal vessels.

All the patients underwent CT simulation, and CT images were used to design the EF-IMRT plan by the same designer. The clinical target volume (CTV) forEF-IMRT included the gross disease, cervix, parametrium, uterus, sufficient vaginal margin from the gross disease (at least $3 \mathrm{~cm}$ ), and regional lymph nodes (presacral, obturator, common iliac, internal iliac, external iliac, and PALNs). Thus, the superior border of target volume for EF-IMRT was placed at the L2-L3 interspace, while the inferior border was at the inferior margin of the obturator foramen but $3 \mathrm{~cm}$ below any palpable disease in the lower vagina. Positive lymph nodes were separately delineated as nodal gross target volume (GTVnd). The CTV and GTVnd with an extra 0.5 - to $0.7-\mathrm{cm}$ margin were used as the planning target volumes (PTVc and PTVn, respectively). The planning constraints of normal tissue were as follows: for the rectum and bladder, < $50 \%$ of the volume received $30 \mathrm{~Gy} ;<30 \%$ of the volume of the head of femur received $30 \mathrm{~Gy}$. The total EFIMRT dose for the PTVc was $45 \mathrm{~Gy}$ in 25 fractions of $1.8 \mathrm{~Gy}$ (1.8 Gy a day, 5 days a week). A simultaneously integrated boost-IMRT (SIB-IMRT) was administered at a dose of 55-60 Gy for lymph node metastasis. We conducted MRI or CT imaging when 22-25 times the EF-IMRT was delivered. If any remaining positive lymph nodes (short-axis diameter, $\geq 5 \mathrm{~mm}$ ) were detected in this examination, a second CT simulation was conducted, and based on the second set of planning images, these lymph nodes were prescribed a sequential boost-IMRT(SeB-IMRT) at a total dose of 8-16 Gy in 4-8 fractions.

High-dose-rate intracavitary brachytherapy (HDR-ICBT) based on CT guidance was administered after EFIMRT once or twice a week, using a remote after-loading system (microSelectron, Nucletron, the Netherlands), which employed an ${ }^{192}$ Ir source for intraoperative planning with real-time dynamic dose calculation. A cumulative HDR-ICBT dose of $12-36$ Gy was prescribed to point A and administered in 2-6 fractions.

In the present study, the biological dose equivalent to 2-Gy fractions (EQD2) was calculated for point A and lymph node metastasis, using the following equation:

$$
E Q D 2=D \times\left[\frac{d+\alpha / \beta}{2+\alpha / \beta}\right]
$$

where $D$ is the total physical dose, $d$ is the physical dose per fraction, and $\alpha / \beta=10$.

\section{Chemotherapy}

Concurrent chemotherapy consisted of weekly cisplatin (40 mg a week). For maintaining continuity of radiation, when myelosuppression grade $\geq 2$ was detected, concurrent chemotherapy was delayed. 
Toxicity was re-evaluated after 1 week, and concurrent chemotherapy was withheld until the white blood cell count, hemoglobin concentration, and platelet count recovered to more than $4 \times 10^{9} / \mathrm{L}$, more than $90 \mathrm{~g} / \mathrm{L}$, and more than $75 \times 10^{9} / \mathrm{L}$, respectively. The adjuvant chemotherapy protocol was as follows:

$135 \mathrm{mg} / \mathrm{m}^{2}$ paclitaxel plus $75 \mathrm{mg} / \mathrm{m}^{2}$ cisplatin every 3 weeks. Patients' informed consents were obtained, and adjuvant chemotherapy was administered after chemoradiation and brachytherapy, based on the patients' overall condition, including their hepatorenal function and marrow function.

\section{Follow-up}

After treatment completion, the patients were required to be re-examined every three months for the first year and every six months for the second year, and every year thereafter. The follow-up exam included gynecological examination, chest X-ray, abdominopelvic MRI, blood biochemistry, SCC-associated antigen, and routine urine and stool examinations. Toxicity was assessed every week during the treatment and at the time of every review check, according to the toxicity criteria of the Radiation Therapy Oncology Group (RTOG) [7].

\section{Statistical analysis}

Overall survival (OS) was defined from the date of treatment initiation to the date of death or to the date of the last follow-up. Disease-free survival (DFS) was defined from completion of treatment to the date of confirming recurrence and metastasis or death. Local control rate (LCR) was defined as the absence of disease at the original site of the tumor on imaging, gynecological examination, or biopsy. Regional control rate (RCR) was defined as absence of lesion in regional lymph node. Distant metastasis-free survival (DMFS) was defined as the beginning of radiotherapy to the detection of distant metastasis or distant metastasis-related death. The OS, DFS, LCR, RCR, and DMFS were calculated with the KaplanMeier method using the statistical software SPSS version 22.0. The obtained values were compared using the log-rank test. The log-rank method was also used to perform univariate analysis when differences between groups were found to be statistically significant $(P<0.05)$. The Cox regression model was used for multivariate analysis. A P value of $<0.05$ was considered statistically significant.

\section{Results}

\section{Patient characteristics}

A total of 34 patients were found to be eligible for analysis in this study. The patients' ages ranged from 42-76 years of age, with a median age of 56 years. Table 1 summarizes the baseline characteristics of the patients in this analysis. 
Table 1

General information of patients.

\begin{tabular}{|c|c|c|c|}
\hline Characteristics & Group definition & No. & Ratio (\%) \\
\hline Age & $\begin{array}{l}\geq 60 \\
<60\end{array}$ & $\begin{array}{l}9 \\
25\end{array}$ & $\begin{array}{l}26.5 \\
73.5\end{array}$ \\
\hline FIGO stage & $\begin{array}{l}\text { IIIC1 } \\
\text { IIIC2 } \\
\text { IVA }\end{array}$ & $\begin{array}{l}15 \\
7 \\
12\end{array}$ & $\begin{array}{l}44.1 \\
20.6 \\
35.3\end{array}$ \\
\hline Tumor size & $\begin{array}{l}\leq 4 \mathrm{~cm} \\
>4 \mathrm{~cm}\end{array}$ & $\begin{array}{l}3 \\
31\end{array}$ & $\begin{array}{l}8.8 \\
91.2\end{array}$ \\
\hline Rectal invasion & $\begin{array}{l}\text { Yes } \\
\text { No }\end{array}$ & $\begin{array}{l}5 \\
29\end{array}$ & $\begin{array}{l}14.7 \\
85.3\end{array}$ \\
\hline Bladder invasion & $\begin{array}{l}\text { Yes } \\
\text { No }\end{array}$ & $\begin{array}{l}11 \\
23\end{array}$ & $\begin{array}{l}32.4 \\
67.6\end{array}$ \\
\hline PLN metastasis & $\begin{array}{l}\text { Yes } \\
\text { No }\end{array}$ & $\begin{array}{l}34 \\
0\end{array}$ & $\begin{array}{l}100.0 \\
0.0\end{array}$ \\
\hline PALN metastasis & $\begin{array}{l}\text { Yes } \\
\text { No }\end{array}$ & $\begin{array}{l}12 \\
22\end{array}$ & $\begin{array}{l}35.3 \\
64.7\end{array}$ \\
\hline Concurrent chemotherapy & $\begin{array}{l}\geq 4 \text { cycles } \\
<4 \text { cycles } \\
\text { None }\end{array}$ & $\begin{array}{l}7 \\
10 \\
17\end{array}$ & $\begin{array}{l}20.6 \\
29.4 \\
50.0\end{array}$ \\
\hline Adjuvant chemotherapy & $\begin{array}{l}\geq 3 \text { cycles } \\
<3 \text { cycles } \\
\text { None }\end{array}$ & $\begin{array}{l}7 \\
7 \\
20\end{array}$ & $\begin{array}{l}20.6 \\
20.6 \\
58.8\end{array}$ \\
\hline Pretreatment HB & $\begin{array}{l}<110 \mathrm{~g} / \mathrm{L} \\
\geq 110 \mathrm{~g} / \mathrm{L}\end{array}$ & $\begin{array}{l}16 \\
18\end{array}$ & $\begin{array}{l}47.1 \\
52.9\end{array}$ \\
\hline EQD2 (point A) & $\begin{array}{l}<70 \mathrm{~Gy} \\
70-80 \mathrm{~Gy} \\
\geq 80 \mathrm{~Gy}\end{array}$ & $\begin{array}{l}2 \\
6 \\
26\end{array}$ & $\begin{array}{l}5.9 \\
17.6 \\
76.5\end{array}$ \\
\hline
\end{tabular}

In terms of radiation, two patients received less than 70 Gy in EQD2 to point A, as they could not endure brachytherapy and refused to finish the whole treatment plan. All the details of the radiation therapy prescribed to point A are summarized in Table 2. 
Table 2

EQD2 of radiation to point $A$.

\begin{tabular}{|lll|}
\hline EQD2 to point A(EF-IMRT + brachytherapy) & No. & Ratio (\%) \\
\hline $60.25 \mathrm{~Gy}(44.25 \mathrm{~Gy}+16 \mathrm{~Gy})$ & 1 & 2.9 \\
\hline $68.25 \mathrm{~Gy}(44.25 \mathrm{~Gy}+24 \mathrm{~Gy})$ & 1 & 2.9 \\
$76.25 \mathrm{~Gy}(44.25 \mathrm{~Gy}+32 \mathrm{~Gy})$ & 6 & 17.6 \\
$84.25 \mathrm{~Gy}(44.25 \mathrm{~Gy}+40 \mathrm{~Gy})$ & 25 & 73.5 \\
\hline $92.25 \mathrm{~Gy}(44.25 \mathrm{~Gy}+48 \mathrm{~Gy})$ & 1 & 2.9 \\
\hline $\begin{array}{l}\text { With regard to metastatic lymph nodes, SIB-IMRT doses ranged from 55-60 Gy. Then, MRI or CT } \\
\text { imaging was carried out to detect the remaining positive lymph nodes (short-axis diameter } \geq 5 \text { mm) } \\
\text { in eight patients after the completion of SIB-IMRT. These patients were prescribed SeB-IMRT (8- }\end{array}$ \\
$\begin{array}{l}\text { 16Gy), which only aimed at the remaining positive lymph nodes. Thus, the total doses for positive } \\
\text { regional lymph nodes were 55-76 Gy (EQD2: 55.92-78 Gy). Table 3 summarizes the details of the } \\
\text { radiation therapy for metastatic lymph nodes. Of the eight patients who were prescribed SeB-IMRT, } \\
\text { five had positive PALNs at diagnosis and the other three had positive PLNs only. }\end{array}$ \\
\hline
\end{tabular}

Table 3

EQD2 of radiation for positive pelvic or para-aortic lymph nodes.

\begin{tabular}{|llll|}
\hline EQD2 for SIB-IMRT & EQD2 for SeB-IMRT & Total EQD2 for positive lymph nodes & N.(\%) \\
\hline 55.92 to $62 \mathrm{~Gy}$ & No nodal boost & 55.92 to $62 \mathrm{~Gy}$ & $26(76.5 \%)$ \\
\hline 55.92 to $62 \mathrm{~Gy}$ & 8 to $16 \mathrm{~Gy}$ & 63.92 to $78 \mathrm{~Gy}$ & $8(23.5 \%)$ \\
\hline
\end{tabular}

Among the 34 patients, 7 received more than 4 cycles of concurrent chemotherapy, 10 received $1-3$ cycles, and 17 patients did not receive any concurrent chemotherapy (Table 1). The reasons for incomplete concurrent chemotherapy (< 4 cycles) included myelosuppression (13 patients), anemia (5 patients), gastrointestinal toxicity (1 patient), and refusing concurrent chemotherapy for personal reasons (8 patients).

Fourteen patients received adjuvant chemotherapy, including seven who received 3 cycles and seven, who received $1-2$ cycles, while 20 patients did not undergo adjuvant chemotherapy.

\section{Treatment Outcomes}

The median follow-up time was 46.5 (range, 6-72) months. Tumor response was assessed using gynecological and imaging examinations three months after treatment completion. Complete response (CR) was defined as the clinical disappearance of all lesions. Of the 34 patients, 31 achieved CR (91.2\%) after primary treatment, and the remaining exhibited persistent or progressive disease $(3 / 34,8.8 \%)$. The 1 - 
and 3-year values of OS, DFS, LCR, RCR, and DMFS were $88.2 \%$ and $73.5 \%, 70.6 \%$ and $70.6 \%, 88.1 \%$ and $88.1 \%, 87.9 \%$ and $87.9 \%, 84.8 \%$ and $81.6 \%$, respectively (Fig. 1 ).

\section{Analysis Of Prognostic Factors}

Relative factors with a $P$ value of $<0.1$ on univariate analysis were included in the multivariate analysis (Table 5). The $\mathrm{P}$ values for pretreatment $\mathrm{HB}$ level, rectal invasion, and PALN metastasis were all $<0.1$ in the univariate analysis for OS (Table 4), while only the pretreatment HB level and PALN metastasis showed statistical significance $(P<0.1)$ for DFS in the univariate analysis (Table 4). Furthermore, only PALN metastasis was of statistical significance in the multivariate analysis, suggesting that PALN metastasis was the independent factor for OS $(P<0.007)$ and DFS $(P<0.005$; Table 5$)$. None of the prognostic factors showed significance with LCR, RCR and DMFS in the multivariate analysis (Table 5), although rectal invasion was significant for RCR $(P<0.1)$ and PALN metastasis was significant for DMFS $(P<0.1)$ in the univariate analysis (Table 4$)$.

Table 4

Univariate analysis for prognostic factors.

\begin{tabular}{|llllll|}
\hline Variable & $\begin{array}{l}\text { OS } \\
\text { P value }\end{array}$ & $\begin{array}{l}\text { DFS } \\
\text { P value }\end{array}$ & $\begin{array}{l}\text { LCR } \\
\text { P value }\end{array}$ & $\begin{array}{l}\text { RCR } \\
\text { P value }\end{array}$ & $\begin{array}{l}\text { DMFS } \\
\text { P value }\end{array}$ \\
\hline Age & 0.748 & 0.593 & 0.945 & 0.908 & 0.524 \\
\hline FIGO stage & 0.100 & 0.265 & 0.902 & 0.707 & 0.574 \\
\hline PALN metastasis & $0.005 \triangle$ & $0.004 \triangle$ & 0.269 & 0.102 & $0.078 \triangle$ \\
\hline Tumor size & 0.516 & 0.493 & 0.681 & 0.674 & 0.597 \\
\hline Rectal invasion & $0.082 \triangle$ & 0.127 & 0.552 & $0.070 \triangle$ & 0.199 \\
\hline Bladder invasion & 0.895 & 0.560 & 0.107 & 0.734 & 0.400 \\
\hline Concurrent chemotherapy & 0.482 & 0.633 & 0.895 & 0.816 & 0.921 \\
\hline Adjuvant chemotherapy & 0.264 & 0.434 & 0.959 & 0.913 & 0.749 \\
\hline Pretreatment HB & $0.045 \triangle$ & $0.032 \triangle$ & 0.144 & 0.306 & 0.945 \\
\hline EQD2 (point A) & 0.713 & 0.803 & 0.946 & 0.281 & 0.520 \\
\hline
\end{tabular}


Table 5

Multivariate analysis for prognostic factors.

\begin{tabular}{|c|c|c|c|}
\hline Subject & HR & $\mathrm{Cl} 95 \%$ & $P$ value \\
\hline \multicolumn{4}{|l|}{ os } \\
\hline PALN metastasis & 9.910 & $1.867-52.598$ & $0.007 \triangle$ \\
\hline Pretreatment HB & 1.567 & $0.347-7.078$ & 0.559 \\
\hline Rectal invasion & 2.613 & $0.561-12.179$ & 0.221 \\
\hline \multicolumn{4}{|l|}{ DFS } \\
\hline PALN metastasis & 9.815 & $1.981-48.629$ & $0.005 \triangle$ \\
\hline Pretreatment HB & 0.149 & $0.011-2.038$ & 0.154 \\
\hline \multicolumn{4}{|l|}{ RCR } \\
\hline Rectal invasion & 6.183 & $0.863-44.311$ & 0.070 \\
\hline \multicolumn{4}{|l|}{ DMFS } \\
\hline PALN metastasis & 4.627 & $0.841-25.440$ & 0.078 \\
\hline \multicolumn{4}{|c|}{$\begin{array}{l}\text { The log-rank test was used to compare the OS of patients with positive PALNs to patients with } \\
\text { negative PALNs, showing significant difference between the two groups }(\mathrm{P}=0.001)(\text { Fig. } 2 \text { ). The three } \\
\text { year OS, DFS, LCR, RCR, and DMFS of patients with and without positive PALNs were } 41.7 \% \text { and } \\
90.9 \%, 33.3 \% \text { and } 90.9 \%, 65.6 \% \text { and } 100 \%, 72.2 \% \text { and } 95.5 \%, 61.9 \% \text { and } 90.9 \% \text {, respectively (Fig. 2- } \\
6 \text { ). }\end{array}$} \\
\hline
\end{tabular}

\section{Patterns Of Treatment Failure}

Three patients experienced persistent or progressive disease (8.8\%). Seven patients $(20.6 \%)$ experienced recurrences. The sites of distant recurrence included lung, para-aortic, mediastinal and supraclavicular lymph nodes, and lung was the most frequent site of recurrences. Four patients experienced regional nodal failure $(4 / 34,11.8 \%)$ : of these, three were PALN recurrence with distant metastasis, and one was both PLN and PALN recurrence with distant metastasis. Table 6 summarizes the information about treatment failures in detail. 
Table 6

Patterns of treatment failure.

\begin{tabular}{|c|c|}
\hline patterns of treatment failure & $n(\%)$ \\
\hline \multicolumn{2}{|l|}{$\begin{array}{l}\text { Patients with positive PALNs } \\
\mathrm{N}=12\end{array}$} \\
\hline local persistent disease & $3(25 \%)$ \\
\hline local recurrence only & $1(8.3 \%)$ \\
\hline distant metastasis only & $1(8.3 \%)$ \\
\hline $\begin{array}{l}\text { PLNs and PALNs recurrence simultaneously } \\
\text { with distant metastasis }\end{array}$ & $1(8.3 \%)$ \\
\hline $\begin{array}{l}\text { PALNs recurrence simultaneously } \\
\text { with distant metastasis }\end{array}$ & $2(16.7 \%)$ \\
\hline \multicolumn{2}{|l|}{$\begin{array}{l}\text { Patients with negative PALNs } \\
N=22\end{array}$} \\
\hline distant metastasis only & $1(4.5 \%)$ \\
\hline $\begin{array}{l}\text { PALNs recurrence simultaneously } \\
\text { with distant metastasis }\end{array}$ & $1(4.5 \%)$ \\
\hline \multicolumn{2}{|c|}{$\begin{array}{l}\text { Of the ten patients who experienced treatment failures, eight had positive PALNs. Four local failures } \\
\text { occurred in patients with positive PALNs. Of these four, three patients had local persistent disease, so } \\
\text { only one patient experienced true local recurrence. Although all the four patients who experienced } \\
\text { local treatment failure received EF-IMRT and } 4-5 \text { fractions of brachytherapy, one received only two } \\
\text { cycles of concurrent chemotherapy and the other three received none. The regional nodal failure rate } \\
\text { for patients with positive PALNs was } 25.0 \%(3 / 12) \text {. Obviously, apart from persistent disease, distant } \\
\text { metastasis with or without regional recurrence }(4 / 12) \text { was the most frequent treatment failure pattern } \\
\text { in patients with positive PALNs. }\end{array}$} \\
\hline
\end{tabular}

Of the 22 patients with negative PALNs during diagnosis, only 2 experienced treatment failure. Both these patients had distant metastasis, and 1 experienced regional nodal failure $(1 / 22,4.5 \%)$.

\section{Treatment-related Toxicity}

Treatment-related toxicity is one major concern for EF-IMRT and nodal dosage boost. We recorded both acute and delayed toxicity data in this analysis. There were no instances of grade 4 acute toxicity and delayed toxicity. All grade 3 acute toxicities observed were hematological toxicities. We found a total of five events $(14.7 \%)$ of grade 3 acute toxicity. With regard to delayed toxicity, only few cases had complications in the urinary and gastrointestinal systems and the rates of $\geq$ grade 3 treatment-related delayed toxicity among patients with and without positive PALNs were $8.3 \%(1 / 12)$ and $4.5 \%(1 / 22)$, respectively. Table 7 summarizes the treatment-related toxicities in this analysis. All these results indicated that EF-IMRT along with dosage boost for lymph node metastasis, concurrent chemotherapy, 
and brachytherapy with or without adjuvant chemotherapy is a safe therapeutic option for treating FIGO IIICr and IVA cervical cancer patients with positive regional lymph nodes.

Table 7

Acute and delayed toxicity after treatment.

\section{Grade 3(Patients without or with positive PALNs) \\ Grade 4(Patients without or with positive PALNs)}

Acute toxicity

\begin{tabular}{lll} 
Gastrointestinal & 0 & 0 \\
\hline Genitourinary & 0 & 0 \\
\hline Leukopenia & $3(2,1)$ & 0 \\
\hline Anaemia & $1(0,1)$ & 0 \\
\hline Thrombocytopenia & $1(1,0)$ & 0 \\
\hline Delayed toxicity & & \\
Gastrointestinal & $1(0,1)$ & 0 \\
\hline Genitourinary & $1(1,0)$ & 0
\end{tabular}

\section{Discussion}

The results of the present study suggested that para-aortic involvement was the strongest predictor of recurrence and a poor prognostic factor for long-term OS and DFS in grades IIICr and IVA cervical cancer patients with regional lymph node metastasis. This finding is consistent with the results reported in previous studies [1]. In a previous study it was reported that there seemed to be a pattern of lymphatic spread among cervical cancer patients from the pelvic to the PALNs and the supraclavicular lymph nodes [14]. Thus, extended-field radiotherapy,which prophylactically addresses the para-aortic region to prevent PALN metastasis, is a feasible strategy to treat stage IIIC1r and IVA cervical cancer patients without PALN metastasis at the time of diagnosis to improve their OS and DFS.

In our study, we implemented EF-IMRT to not only prevent but also to treat PALN metastasis. Additionally, SIB-IMRT and SeB-IMRT were delivered to treat all positive regional lymph nodes. Currently, compared with the conventional techniques, IMRT could reduce the incidence of treatment-related toxicities when delivering extended-field radiotherapy and nodal boost to treat lymph node metastasis [4]. SIB and SeB were two delivery methods of IMRT boost, but most studies concentrating on IMRT boost only implemented one of these modalities, and very few studies have utilized both SIB and SeB to treat nodal metastasis [12]. In the present study, 22 patients with stage IIIC1 $r$ and IVA cervical cancer and without positive PALNs were administered prophylactic EF-IMRT, and 12 patients with stage IIIC2r and IVA cervical cancer and with positive PALNs were administered definitive EF-IMRT. Regional positive lymph nodes 
found in all 34 patients were treated with SIB-IMRT, and 8 received SeB-IMRT for the remaining positive lymph nodes according to CT/MRI conducted at the end of SIB-IMRT. Feng et al. [11] indicated that SeBIMRT would extend the total treatment time and also increase the treatment-related toxicity due to the additional boost, which delivers an increased dose to the surrounding normal structures. In our study, the SeB-IMRT irradiation fields were very small, as it was only aimed at the remaining positive regional lymph nodes (short-axis diameter $\geq 5 \mathrm{~mm}$ ) after SIB-IMRT. Due to the small irradiation fields of SeB-IMRT, the planning process for SeB-IMRT was simple, with limited treatment-related toxicity; thus, brachytherapy was carried out simultaneously with SeB-IMRT. Therefore, SeB-IMRT did not increase the treatment time in our study and the resultant adverse events were also of acceptable levels.

In the negative PALN cohort in our study, the 3-year OS, DFS, RCR, and rate of $\geq$ grade 3 delayed toxicity were $90.9 \%, 90.9 \%, 95.5 \%$, and $4.5 \%$, respectively. Liang et al. [16]enrolled 47 patients with positive PLNs (negative PALNs) and delivered extended-field external beam radiation therapy and nodal boost via 2-or 3-dimensional technique, and reported a $54 \%$ DFS, $62 \%$ OS, $51 \%$ RCR, and $11 \%$ accumulative rate of $\geq$ grade 3 treatment-related late toxicity at 3 years. Obviously, compared with the conventional technique mentioned in the above study, our study had improved survival and lower treatment-related toxicity. Furthermore, our outcomes of the negative PALN cohort were comparable with the study of Vargo et al., which delivered EF-IMRT and SIB-IMRT to 41 patients without positive PALNs and achieved a 3-year OS, DFS, RCR, and rate of $\geq$ grade 3 delayed toxicity of $73 \%, 64 \%, 95 \%$, and $4 \%$, respectively [4]. In this comparison, the OS and DFS were improved and the rate of treatment-related toxicity was low with addition of SeB-IMRT in the nodal dosage boost. The favorable results of the present study suggested that EF-IMRT and nodal dosage boost were safe and could control the involved PLNs and improve the OS and DFS for stage IIIC1r and IVA patients with positive PLNs (negative PALNs).

In the positive PALNs cohort in our study, the 3-year OS, DFS, RCR, and rate of $\geq$ grade 3 delayed toxicity were $41.7 \%, 33.3 \%, 72.2 \%$, and $8.3 \%$, respectively. In arm 1 of the RTOG 0116 trial [15], 26 patients with positive PALNs were treated with extended-field radiation and nodal dosage boost via conventional technology, and the DFS, OS, RCR, and rate of $\geq$ grade 3 treatment-related toxicity at 1.5 years were reported to be $46 \%, 60 \%, 54 \%$, and $40 \%$, respectively. Obviously, the RCR and rate of delayed toxicity for the positive PALN cohort treated with definitive EF-IMRT showed favorable results in comparison with the historic control from the classic cooperative group data of cases treated with non-IMRT techniques. Additionally, Vargo et al. [4] analyzed 20 patients with positive PALNs treated with definitive EF-IMRT along with SIB-IMRT and reported a better 3-year RCR, DFS, OS, and rate of late $\geq$ grade 3 toxicity $(89 \%$, $40 \%, 61 \%$, and 0 respectively) compared to the results obtained in our present study $(72.7 \%, 33.3 \%, 41.7 \%$, and $8.3 \%$ respectively). However, our study had more IVA patients $(5 / 12,42 \%)$ than the abovementioned study $(5 / 61,8 \%)$. As previous studies reported that stage IVA is a poor prognostic factor with a high risk of PALN involvement $[17,18]$, stage IVA patients are more likely to experience treatment failure. The rate of $\geq$ grade 3 treatment-related delayed toxicity was found to be higher in our positive PALN cohort (8.3\%) than in the report of Vargo et al. ( $0 \%)$, but only one patient (1/12) in the positive PALN cohort developed grade 3 treatment-related delayed toxicity. Our results from the positive PALNs cohort suggested that EF- 
IMRT and nodal dosage boost were well tolerated by the patients and could eradicate lesions in involved PLNs and PALNs for IIIC2r and IVA patients with positive PALNs.

Although stage IIIC2r and IVA patients with positive PALNs had better RCR in our study than in similar reports in previous studies utilizing traditional techniques, the OS and DFS were unsatisfactory compared to arm 1 of the RTOG 0116 trial [15]. PALN involvement was considered to be regional metastasis in the 2018 version of the FIGO staging system, indicating that regional treatment could still be implemented for PALN-positive patients without distant metastasis. Among the 12 grades IIIC2 $\mathrm{r}$ and IVA patients with positive PALNs, the 1-year and 3-year OS, LCR, and DFS were $66.7 \%$ and $41.7 \%, 65.6 \%$ and $65.6 \%$, and $33.3 \%$ and $33.3 \%$, respectively. These poor survival rates may probably be due to distant failure $(4 / 12)$ and local failure (4/12). Four local failures were recorded in four of the twelve patients with PALN metastasis, and all four patients did not receive adequate concurrent chemotherapy. Concurrent chemotherapy is considered an important treatment strategy for LACC, reducing the local and distant recurrences and improving the survival for LACC [9]. Although prognostic factor analysis for OS, DMFS, and LCR did not show statistical significance in concurrent chemotherapy in our study (Table 3), insufficiency of concurrent chemotherapy might partly explain why patients with PALN metastasis in our study had low OS and LCR.

Apart from local failure, there were three PALN failures and four distant failures, leading to 3-year RCR and DMFS values of $72.2 \%$ and $60.2 \%$. These figures were significantly lower than those in PALNnegative patients in the present study, suggesting that PALN metastasis was more than a regional disease. Moreover, PALN disease was considered as stage IVB in several studies $[8,19]$. As we know, systemic chemotherapy is the crucial treatment to control dissemination in stage IVB cervical cancer, so adjuvant chemotherapy (ACT) may be a rational therapy to improve the OS of stage IIIC2 and IVA patients with positive PALNs. But four recent randomized controlled trials (RCTs) designed to compare concurrent chemoradiation therapy(CCRT) alone and CCRT followed by ACT showed two inconsistent conclusions about adjuvant chemotherapy [20-23]. Two of the four RCTs showed that patients with LACC got significant survival benefits from ACT $[20,21]$ whereas the other two trials concluded the opposite [22, 23]. The effect of ACT on LACC was unclear but it is worth noting that 2 or 3 cycles of cisplatin plus paclitaxel or gemcitabine were administered as adjuvant chemotherapyto most patients in the four RCTs mentioned above. Based on the view that PALN disease was more advanced than a regional metastasis but a type of distant disease, the addition of adjuvant chemotherapy cycles or adjusting the adjuvant chemotherapy regimen may be one way to improve the OS of stage IIIC2 and IVA patients with positive PALNs. Future studies are needed to address effective systemic therapy for stage IIIC2 $r$ and IVA cervical cancer patients with and without PALN metastasis.

\section{Conclusion}

The results of our retrospective analysis confirmed that PALN metastasis was an independent factor for OS and DFS of cervical cancer patients with FIGO IIICr and IVA with positive regional lymph nodes ( $\mathrm{P}=$ 0.007 and $P=0.005$, respectively). We aimed to treat regional lymph node metastasis in these patients 
using intensive nodal boost to the positive lymph nodes, which consisted of SIB-IMRT and SeB-IMRT. It should be noted that SeB-IMRT was only applied to the lymph nodes (short-axis diameter $\geq 5 \mathrm{~mm}$ ) that were detected positive in the CT or MRI imaging carried out after the completion of SIB-IMRT. Overall, our results suggested that EF-IMRT and intensive nodal boost were safe, with acceptable levels of treatmentrelated toxicity, and improved the RCR of IIICr and IVA cervical cancer patients with positive regional lymph nodes. Moreover, EF-IMRT and intensive nodal boost significantly improved the OS and DFS for IIIC1 $r$ and IVA patients with negative PALNs (positive PLNs). To improve the survival of patients with grades IIIC2 and IVA cervical cancer with positive PALNs, adjustment of adjuvant chemotherapy cycles or regimen might be considered.

\section{Declarations}

\section{List of abbreviations}

CCRT Concurrent chemoradiation therapy

$\mathrm{CR} \quad$ Complete response

CT Computed tomography

CTV Clinical target volume

DFS Disease-free survival

DMFS Distant metastasis-free survival

EBRT External beam radiation therapy

ECOG Eastern Cooperative Oncology Group

FDG-PET 18-fluorodeoxyglucose positron emission tomography

FIGO Federation of Gynecology and Obstetrics

IMRT Intensity-modulated radiotherapy

LACC Locally advanced cervical cancer

LCR Local control rate

MRI Magnetic resonance imaging

OS Overall survival

PALN Para-aortic lymph node 
PLN Pelvic lymph node

QOL Quality of life

RCR Regional control rate

SeB Sequential boost

SIB Simultaneously integrated boost

\section{Acknowledgements}

We would like to thank all the patients involved in this study for sharing their information during the follow-up period. We are also very grateful to all the members of our department for the helpful discussions.

\section{Funding}

All the authors declare that this work has not received any funding.

\section{Availability of data and materials}

The datasets used and/or analyzed during the current study are available upon reasonable request.

\section{Authors' contributions}

LG conceived and designed the study.YZ and GL conducted patient's follow-up, data collection, and data analysis.YZ prepared the figuures and wrote the manuscript. All authors read and approved the final manuscript.

\section{Author details}

${ }^{1}$ Graduate student of grade 2018, Guangzhou University of Chinese Medicine, No.232, Waihuandong Road, University Town, Panyu District, Guangzhou, Guangdong, China.

${ }^{2}$ Radiation Oncology Department, Guangzhou University of Chinese Medicine Second Affiliated Hospital (Guangdong Provincial Hospital of Chinese Medicine), No.55, Neihuanxi Road, University Town, Panyu District, Guangzhou, Guangdong, China.

\section{Ethics approval and consent to participate}

All procedures performed in the studies involving human participants were in accordance with the ethical standards of the institutional and/or national research committee and with the 1964 Helsinki declaration and its later amendments or comparable ethical standards. This study was approved by the Ethics Committee of Guangzhou University of Chinese Medicine. 


\section{Consent for publication}

Not applicable.

\section{Competing interests}

The authors declare that they have no competing interests.

\section{References}

1. Creasman, W. T., \& Kohler, M. F. (2004). Is lymph vascular space involvement an independent prognostic factor in early cervical cancer?. Gynecologic oncology, 92(2), 525-529.

2. Lee, J., Lin, J. B., Chang, C. L., Jan, Y. T., Sun, F. J., Wu, M. H., \& Chen, Y. J. (2017). Prophylactic lower para-aortic irradiation using intensity-modulated radiotherapy mitigates the risk of para-aortic recurrence in locally advanced cervical cancer: a 10-year institutional experience. Gynecologic oncology, 146(1), 20-26.

3. Koh, W. J., Abu-Rustum, N. R., Bean, S., Bradley, K., Campos, S. M., Cho, K. R., ... \& Crispens, M. A. (2019). Cervical cancer, version 3.2019, NCCN clinical practice guidelines in oncology. Journal of the National Comprehensive Cancer Network, 17(1), 64-84.

4. Vargo, J. A., Kim, H., Choi, S., Sukumvanich, P., Olawaiye, A. B., Kelley, J. L., ... \& Beriwal, S. (2014). Extended field intensity modulated radiation therapy with concomitant boost for lymph node-positive cervical cancer: analysis of regional control and recurrence patterns in the positron emission tomography/computed tomography era. International Journal of Radiation Oncology* Biology* Physics, 90(5), 1091-1098.

5. Tseng, J. Y., Yen, M. S., Twu, N. F., Lai, C. R., Horng, H. C., Tseng, C. C., ... \& Juang, C. M. (2010). Prognostic nomogram for overall survival in stage IIB-IVA cervical cancer patients treated with concurrent chemoradiotherapy. American journal of obstetrics and gynecology, 202(2), 174-e1.

6. Huang, E. Y., Wang, C. J., Chen, H. C., Fang, F. M., Huang, Y. J., Wang, C. Y., \& Hsu, H. C. (2008). Multivariate analysis of para-aortic lymph node recurrence after definitive radiotherapy for stage IBIVA squamous cell carcinoma of uterine cervix. International Journal of Radiation Oncology* Biology* Physics, 72(3), 834-842.

7. Cox, J. D., Stetz, J., \& Pajak, T. F. (1995). Toxicity criteria of the radiation therapy oncology group (RTOG) and the European organization for research and treatment of cancer (EORTC). International Journal of Radiation Oncology• Biology• Physics, 31(5), 1341-1346区

8. Waggoner, S. E. (2003). Cervical cancer. The Lancet, 361(9376), 2217-2225.

9. Rose, P. G., Bundy, B. N., Watkins, E. B., Thigpen, J. T., Deppe, G., Maiman, M. A., ... \& Insalaco, S. (1999). Concurrent cisplatin-based radiotherapy and chemotherapy for locally advanced cervical cancer. New England Journal of Medicine, 340(15), 1144-1153.

10. Small, W., Winter, K., Levenback, C., lyer, R., Hymes, S. R., Jhingran, A., ... \& Greven, K. (2011).

Extended-field irradiation and intracavitary brachytherapy combined with cisplatin and amifostine for 
cervical cancer with positive para-aortic or high common iliac lymph nodes: results of arm II of Radiation Therapy Oncology Group (RTOG) 0116. International Journal of Gynecologic Cancer, 21(7), 1266-1275.

11. Feng, C. H., Hasan, Y., Kopec, M., \& Al-Hallaq, H. A. (2016). Simultaneously integrated boost (SIB) spares OAR and reduces treatment time in locally advanced cervical cancer. Journal of applied clinical medical physics, 17(5), 76-89.

12. Boyle J, Craciunescu O, Steffey B, Cai J, Chino J. Methods, safety, and early clinical outcomes of dose escalation using simultaneous integrated and sequential boosts in patients with locally advanced gynecologic malignancies. Gynecologic oncology. 2014;135:239-43.

13. Klopp AH, Yeung AR, Deshmukh S, Gil KM, Wenzel L, Westin SN. A phase III randomized trial comparing patient-reported toxicity and quality of life (QOL) during pelvic intensity modulated radiation therapy as compared to conventional radiation therapy. Int J Radiat Oncol Biol Phys. 2016;96:S3.

14. Liang JA, Chen SW, Chang WC, Hung YC, Yeh LS, Yang SN, et al. Risk stratification for failure in patients with advanced cervical cancer after concurrent chemoradiotherapy: another way to optimise treatment results. Clinical oncology. 2008;20:683-90.

15. Small Jr W, Winter K, Levenback C, lyer R, Gaffney D, Asbell S, et al. Extended-field irradiation and intracavitary brachytherapy combined with cisplatin chemotherapy for cervical cancer with positive para-aortic or high common iliac lymph nodes: results of ARM 1 of RTOG 0116. International Journal of Radiation Oncology* Biology* Physics. 2007;68:1081-7.

16. Liang JA, Chen SW, Hung YC, Yeh LS, Chang WC, Lin WC, et al. Low-dose, prophylactic, extendedfield, intensity-modulated radiotherapy plus concurrent weekly cisplatin for patients with stage IB2IIIB cervical cancer, positive pelvic lymph nodes, and negative para-aortic lymph nodes. International Journal of Gynecologic Cancer. 2014;24:901-7.

17. Vandeperre A, Van Limbergen E, Leunen K, Moerman P, Amant F, Vergote I. Para-aortic lymph node metastases in locally advanced cervical cancer: Comparison between surgical staging and imaging. Gynecologic oncology. 2015;138:299-303.

18. Gouy S, Morice P, Narducci F, Uzan C, Martinez A, Rey A, et al. Prospective multicenter study evaluating the survival of patients with locally advanced cervical cancer undergoing laparoscopic para-aortic lymphadenectomy before chemoradiotherapy in the era of positron emission tomography imaging. J Clin Oncol. 2013;31:3026-33.

19. Jung J, Park G, Kim YS. Definitive extended-field intensity-modulated radiotherapy with chemotherapy for cervical cancer with para-aortic nodal metastasis. Anticancer research. 2014; 34: 4361-66.

20. Duenas-González A, Zarbá JJ, Patel F, Alcedo JC, Beslija S, Casanova L, et al. Phase III, open-label, randomized study comparing concurrent gemcitabine plus cisplatin and radiation followed by adjuvant gemcitabine and cisplatin versus concurrent cisplatin and radiation in patients with stage IIB to IVA carcinoma of the cervix. Journal of Clinical Oncology. 2011;29:1678-85. 
21. Tang J, Tang Y, Yang J, Huang S. Chemoradiation and adjuvant chemotherapy in advanced cervical adenocarcinoma. Gynecologic oncology. 2012;125: 297-302.

22. Lorvidhaya V, Chitapanarux I, Sangruchi S, Lertsanguansinchai P, Kongthanarat $Y$, Tangkaratt $S$, et al. Concurrent mitomycin C, 5-fluorouracil, and radiotherapy in the treatment of locally advanced carcinoma of the cervix: a randomized trial. International Journal of Radiation Oncology* Biology* Physics. 2003;55:1226-32.

23. Tangjitgamol S, Tharavichitkul E, Tovanabutra C, Rongsriyam K, Asakij T, Paengchit K, et al. A randomized controlled trial comparing concurrent chemoradiation versus concurrent chemoradiation followed by adjuvant chemotherapy in locally advanced cervical cancer patients: ACTLACC trial. Journal of gynecologic oncology. 2019;30:e82.

24. Bhatla N, Aoki D, Sharma DN, Sankaranarayanan R. Cancer of the cervix uteri. International Journal of Gynecology \& Obstetrics. 2018;143:22-36.

\section{Figures}




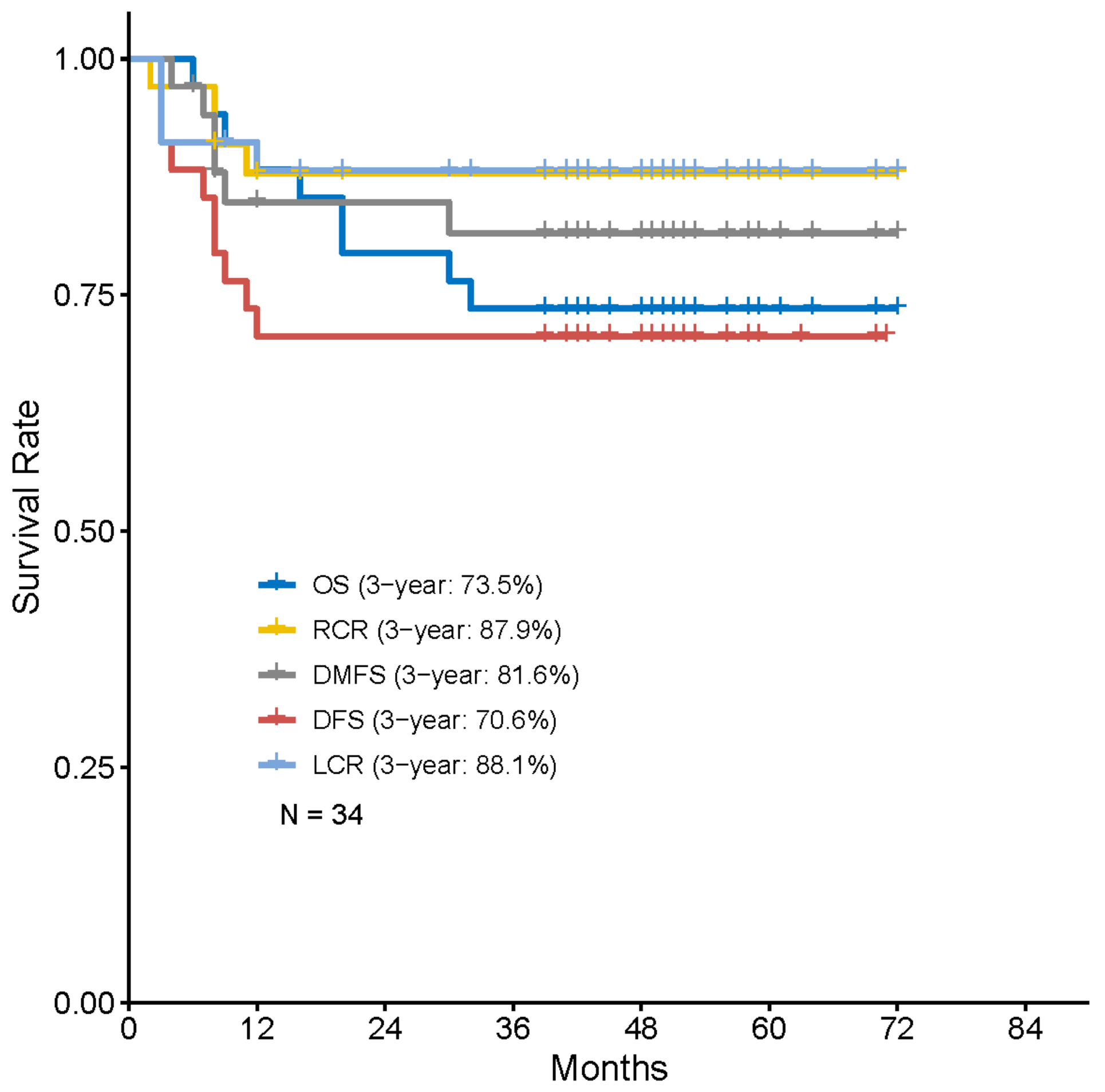

\section{Figure 1}

Kaplan-Meier survival curves for overall survival (OS), regional control rate (RCR), distant metastasis-free survival (DMFS), disease-free survival (DFS), and local control rate(LCR) at three years. 


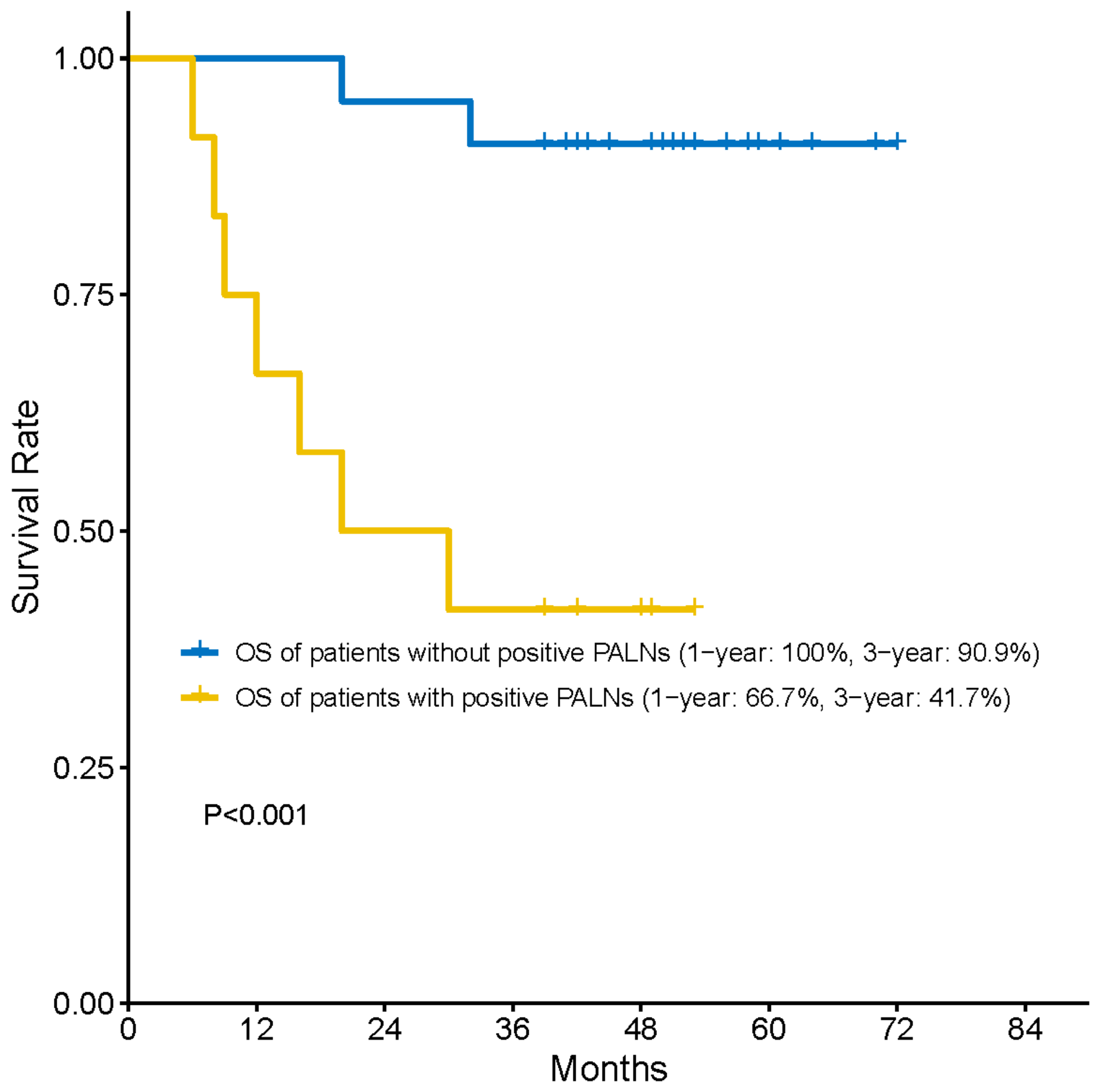

Figure 2

Kaplan-Meier curve demonstrating the OS of patients with and without positive PALNs. 


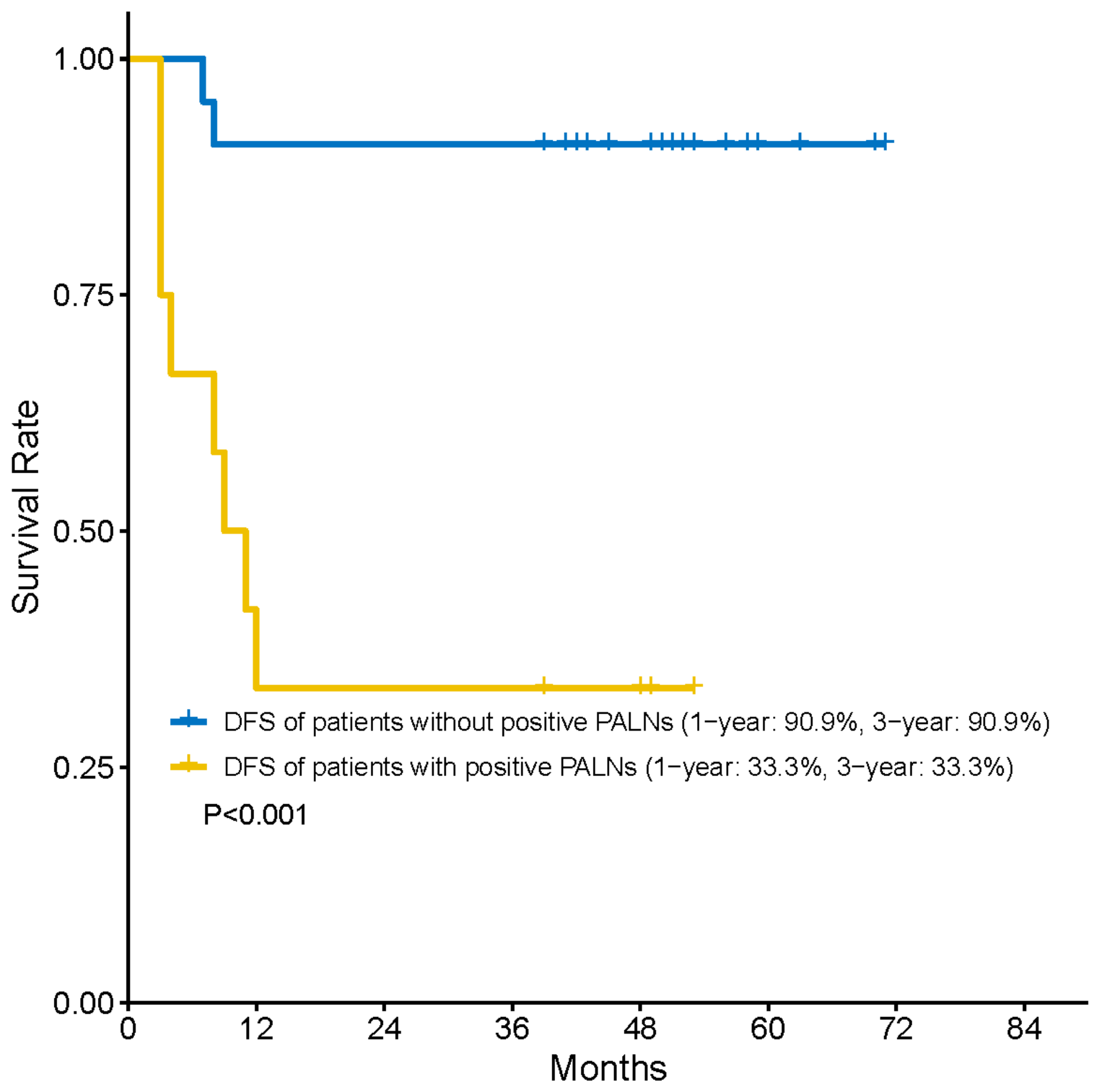

Figure 3

Kaplan-Meier curve demonstrating the DFS of patients with and without positive PALNs. 


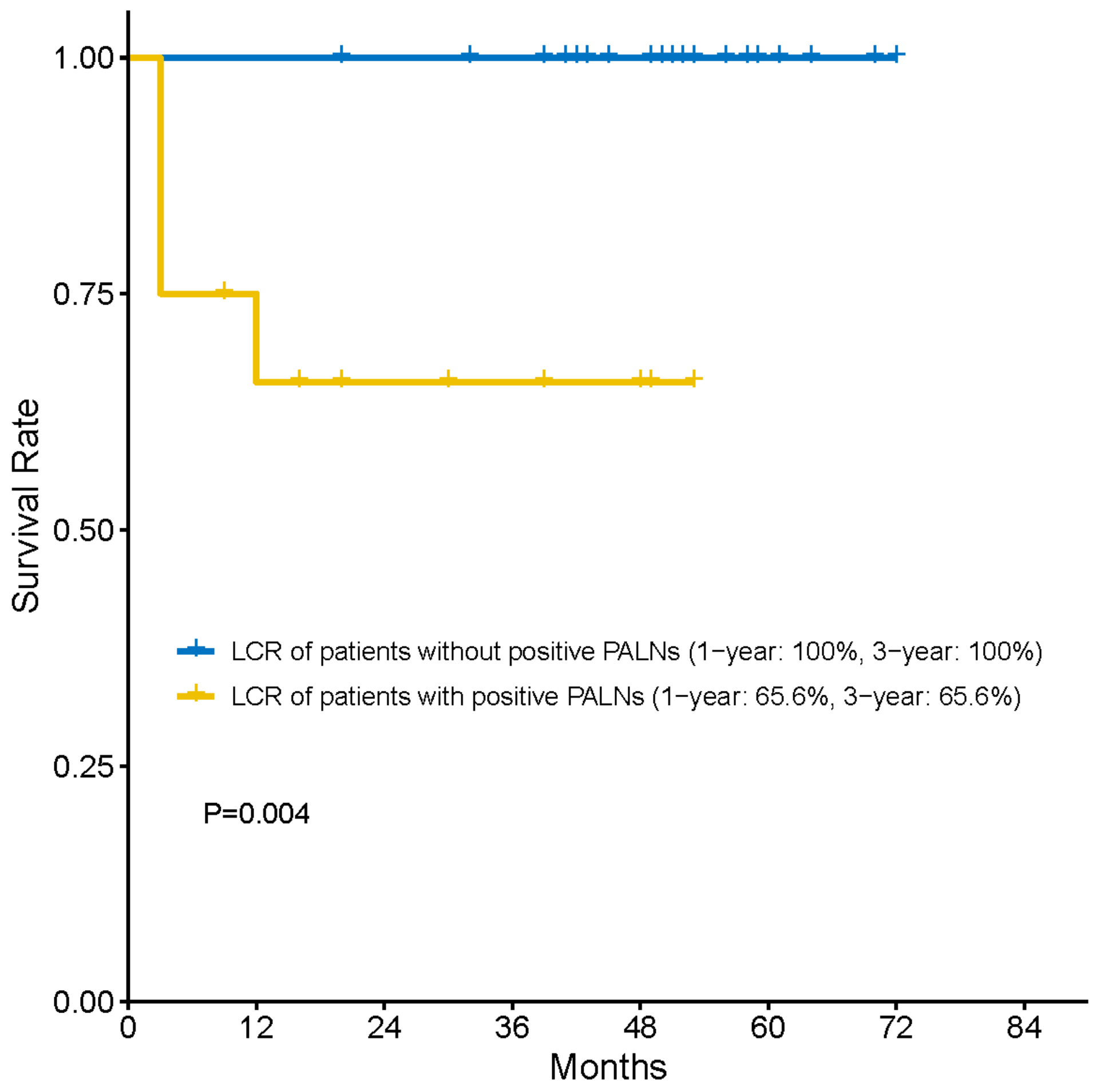

Figure 4

Kaplan-Meier curve demonstrating the LCR of patients with and without positive PALNs. 


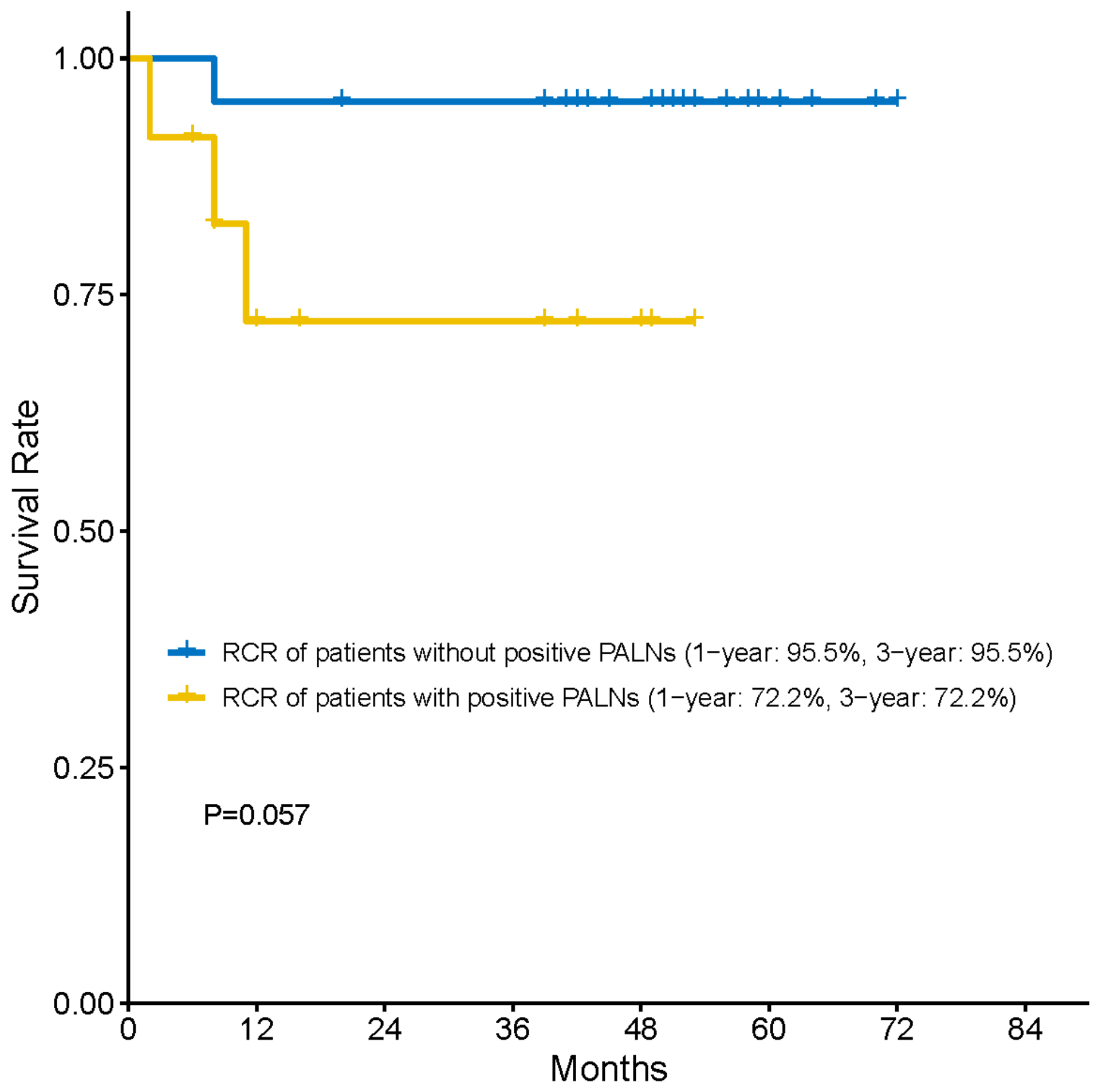

Figure 5

Kaplan-Meier curve demonstrating the RCR of patients with and without positive PALNs. 


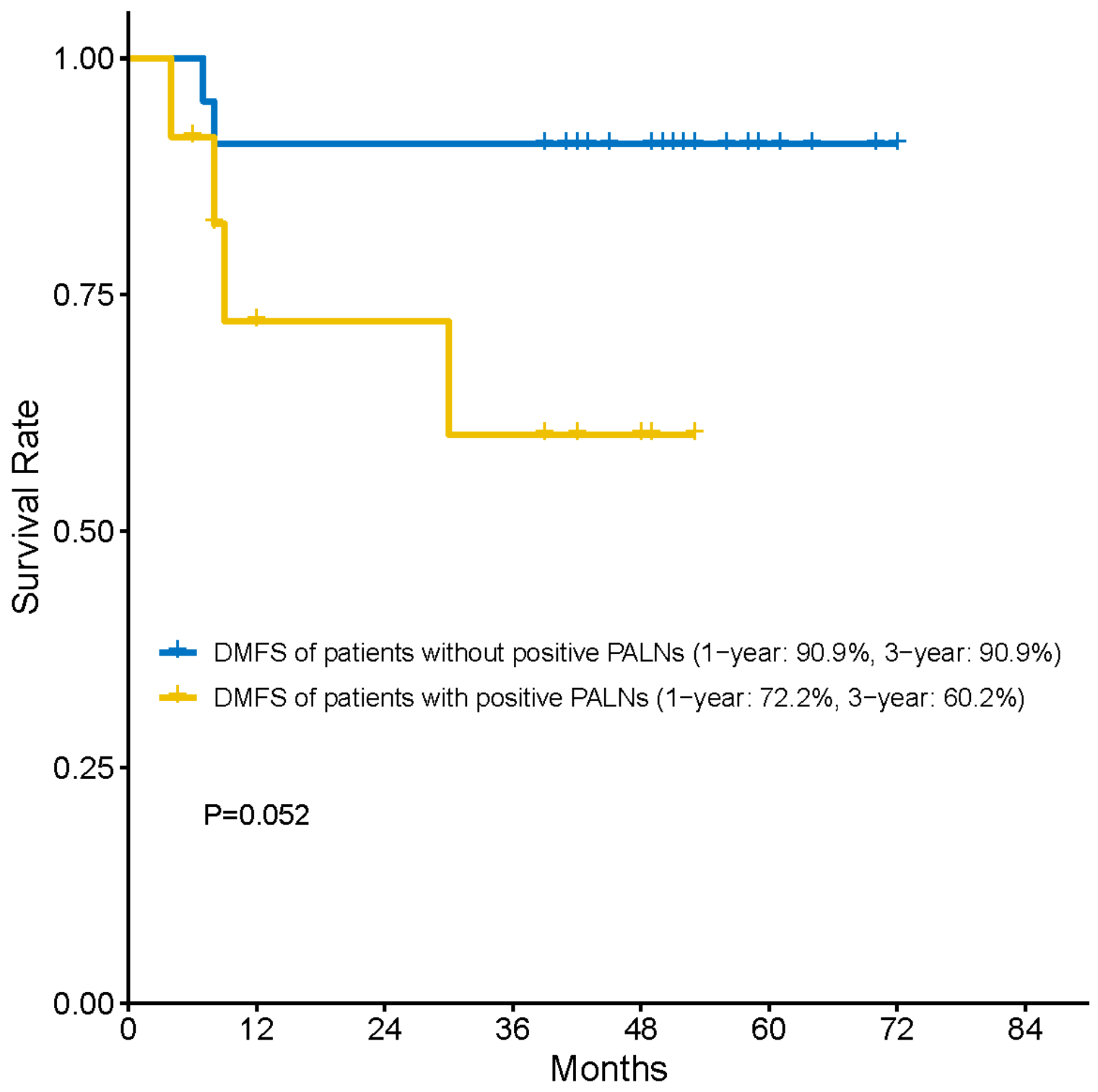

Figure 6

Kaplan-Meier curve demonstrating the DMFS of patients with and without positive PALNs. 